\title{
A comparison of femoral/sciatic nerve block with lateral femoral cutaneous nerve block and combined spinal epidural anesthesia for total knee replacement arthroplasty
}

\author{
Jong Hae Kim ${ }^{1}$, Myoung Rae $\mathrm{Cho}^{2}$, Si Oh Kim³ ${ }^{3}$ Jung Eun Kim ${ }^{1}$, Dong Keun Lee ${ }^{1}$, and Woon Seok Roh ${ }^{1}$ \\ Departments of ${ }^{1}$ Anesthesiology and Pain Medicine, ${ }^{2}$ Orthopedic Surgery, School of Medicine, Catholic University of Daegu, \\ ${ }^{3}$ Department of Anesthesiology and Pain Medicine, Kyungpook National University School of Medicine, Daegu, Korea
}

Background: Several factors, such as compromised cardiopulmonary function, anticoagulative therapy, or anatomical deformity in the elderly, prevent general anesthesia and neuraxial blockade from being conducted for total knee replacement arthroplasty (TKRA). We investigated the efficacy of femoral/sciatic nerve block with lateral femoral cutaneous nerve block (FSNB) as an alternative procedure in comparison with combined spinal epidural nerve block (CSE) in patients undergoing TKRA.

Methods: In this observational study, 80 American Society of Anesthesiologists physical status I-III patients scheduled for elective unilateral TKRA underwent CSE $(n=40)$ or FSNB $(n=40)$. Perioperative side effects, intraoperative medications, duration and remaining amount of intravenous patient-controlled analgesia, rate of satisfaction with the surgical anesthesia and postoperative analgesia, willingness to recommend the same surgical anesthesia and postoperative analgesia to others, and postoperative visual analog scale pain scores were assessed. Statistical analysis was done using Chi-square test, Student's t-test, and repeated-measures analysis of variances.

Results: There was significantly more use of antihypertensives, analgesics, and sedatives in the FSNB group. There were no significant differences of perioperative side effects, duration and remaining amount of intravenous patientcontrolled analgesia, rate of satisfaction with the surgical anesthesia and postoperative analgesia, willingness to recommend the same surgical anesthesia and postoperative analgesia to others, and postoperative visual analog scale scores between the two groups.

Conclusions: FSNB with a sophisticated use of antihypertensives, analgesics, and sedatives to supplement insufficient block offers a practical alternative to CSE for TKRAs. (Korean J Anesthesiol 2012; 62: 448-453)

Key Words: Epidural anesthesia, Femoral nerve, Nerve block, Sciatic nerve, Spinal anesthesia, Total knee replacement.

Received: May 20, 2011. Revised: 1st, July 12, 2011; 2nd, September 13, 2011. Accepted: September 22, 2011.

Corresponding author: Woon Seok Roh, M.D., Ph.D., Department of Anesthesiology and Pain Medicine, School of Medicine, Catholic University of Daegu, 3056-6, Daemyeong-4dong, Nam-gu, Daegu 705-718, Korea. Tel: 82-53-650-4504, Fax: 82-53-650-4517, E-mail: usno@cu.ac.kr (c) This is an open-access article distributed under the terms of the Creative Commons Attribution Non-Commercial License (http:// creativecommons.org/licenses/by-nc/3.0/), which permits unrestricted non-commercial use, distribution, and reproduction in any medium, provided the original work is properly cited. 


\section{Introduction}

Combined spinal epidural anesthesia (CSE) is now in widespread use because it can reduce or eliminate some of the disadvantages of spinal and epidural anesthesia while also preserving their advantages. It offers the speed of onset, efficacy, and minimal toxicity of a spinal block combined with the potential of improving an inadequate block or prolonging the duration of anesthesia with epidural supplements, and extending the analgesia into postoperative period [1]. These advantages make the CSE useful to surgical anesthesia in total knee replacement arthroplasty (TKRA). However, patients undergoing TKRA are usually elderly, and they often have concomitant diseases that compromise cardiovascular and pulmonary function and spinal stability. On occasion, the anatomical deformity caused by a degenerative change of the vertebral column or by morbid obesity, the uncompensated hemodynamic response to CSE-induced physiologic changes resulting from severely decreased cardiac function, and the antiplatelet and anticoagulant therapy preventing ischemic cardiac events make CSE inappropriate to surgical anesthesia for TKRA [2]. These factors even prevent general anesthesia practices from being conducted [3]. With the presence of these factors, alternative anesthetic techniques are needed to perform TKRA. There are many reports about femoral/sciatic nerve block as a postoperative analgesia after TKRA, but few reports about femoral/sciatic nerve block as surgical anesthesia. We therefore compared the efficacy of femoral/sciatic nerve block with lateral femoral cutaneous nerve block (FSNB) and CSE during the perioperative period.

\section{Materials and Methods}

After obtaining approval from the institutional review board, and informed consent, the observational study was carried out on 84 consecutive patients undergoing elective unilateral TKRA. The anesthetic technique used was based on surgeon, anesthesiologist, and/or patient preference. All TKRAs were performed under CSE $(n=42)$ or FSNB $(n=42)$.

In the CSE group, the epidural space below L2 was identified using a loss of resistance to saline. Next, an extra long 27-gauge pencil-point spinal needle was introduced through the Tuohy needle. When correct placement of the spinal needle had been confirmed by free flow of cerebrospinal fluid (CSF), $1.3 \mathrm{ml}$ of $0.5 \%$ hyperbaric bupivacaine was injected, and the spinal needle was then withdrawn. After withdrawing the spinal needle, $10 \mathrm{ml}$ of $0.75 \%$ ropivacaine was injected, and a catheter was introduced, about $4-5 \mathrm{~cm}$ into the epidural space through the Tuohy needle.

In the FSNB group, femoral nerve block was performed using Winnie's technique. Following contraction of the quadriceps femoris muscle and movement of the patella with 0.5 $\mathrm{mA}$ at $1 \mathrm{~Hz}$ using nerve stimulator, $20 \mathrm{ml}$ of $1.5 \%$ mepivacaine were injected. The lateral femoral cutaneous nerve block was performed by the injection of $5 \mathrm{ml}$ of $1.5 \%$ mepivacaine, $2.5 \mathrm{~cm}$ medial, and $2.5 \mathrm{~cm}$ caudal to the anterior superior iliac spine. The sciatic nerve block was based on Labat's technique. After contractions of the gastrocnemius (foot plantar flexion) and/ or tibialis anterior (foot dorsi-flexion) using a stimulus of 0.5 $\mathrm{mA}$ at $1 \mathrm{~Hz}, 20 \mathrm{ml}$ of $1.5 \%$ mepivacaine were injected. Urinary catheters were placed in all patients and were continued until 48 hours postoperatively.

In the event of an incomplete blockade, intravenous injection of $30 \mathrm{mg}$ of ketorolac, $100 \mu \mathrm{g}$ of fentanyl, and a continuous infusion of propofol ( $3 \mathrm{mg} / \mathrm{kg} / \mathrm{hr}$ ) with $1 \mathrm{mg} / \mathrm{kg}$ of bolus were used sequentially for additional analgesia as common practice in our institution. If systolic blood pressure and heart rate were decreased below $100 \mathrm{mmHg}$ and $50 \mathrm{bpm}, 10 \mathrm{mg}$ of ephedrine and $0.5 \mathrm{mg}$ of atropine were administered, respectively. In the event of shivering, 25 to $50 \mathrm{mg}$ of meperidine was administered intravenously, and intravenous administration of ondansetron $(4-8 \mathrm{mg})$ or ramosetron $(0.1-0.2 \mathrm{mg})$ was used when the patient complained of nausea or vomiting. At the end of surgery, the epidural catheter in the CSE group was removed, and intravenous patient-controlled analgesia (IV PCA) composed of 1,000 $\mathrm{g}$ g of fentanyl, $150 \mathrm{mg}$ of ketorolac, and $0.3 \mathrm{mg}$ of ramosetron, with a total volume of $100 \mathrm{ml}$, was initiated for postoperative analgesia, without any supplemental blockades, and was maintained for 48 hours. And the IV PCA settings were a bolus of $1 \mathrm{ml}$, a lockout interval of 15 minutes with no limit, and $2 \mathrm{ml} / \mathrm{hr}$ of background infusion.

For each group, time from the start of drape for anesthetic procedure to the end of local anesthetic injection (preparation time), duration of surgery and time elapsed from the end of local anesthetic injection to the end of the surgery (total anesthetic time) were described. In addition, intraoperative side effects, such as hypotension (systolic blood pressure $<100$ $\mathrm{mmHg}$ ), bradycardia $(<50 \mathrm{bpm})$, shivering, nausea/vomiting, for example, and frequency of use of intraoperative medications for managing these side effects were also described. Postoperatively, all the patients were tracked to determine the time lasting from the end of local anesthetic injection to complete resolution of sensory and motor block (complete resolution time), the rate of satisfaction with the surgical anesthesia and the postoperative analgesia, where 0 means extremely dissatisfied and 100 means extremely satisfied, the willingness to recommend the same surgical anesthesia and postoperative analgesia to others, duration of IV PCA use, and remaining amount of IV PCA. And visual analog scale (VAS) pain scores, where 0 indicates "no pain" and 10 indicates 
"strongest pain imaginable", and postoperative side effects (dizziness, sedation, postoperative nausea/vomiting, and pruritus) were assessed simultaneously 1, 2, 6, 12, 24, and 48 hours after surgery.

Statistical analyses were performed using SPSS software (version 14.0, SPSS Inc, IL, USA). All data are expressed mean \pm SEM or number of patients. Student two tailed t-tests were used to compare the rate of satisfaction with the surgical anesthesia and the postoperative analgesia and other quantitative variables such as age, height, weight, a dose of medications, durations, and complete resolution time between the two groups. The willingness to recommend the same surgical anesthesia and postoperative analgesia to others, intraoperative and postoperative side effects, the frequency of use of intraoperative medications, and other categorical variables were analyzed using the Pearson's Chi-square test. The statistical significance of differences between the VAS scores at the different time points was tested, first using repeated-measures analysis of variance, and, then, with post hoc Student two tailed t-tests where appropriate, using the Bonferroni correction for multiple comparisons. A significant difference was acknowledged if the probability of a type 1 error was $<5 \%$ (i.e., $\mathrm{P}<0.05$ ).

\section{Results}

Eighty-four patients were included in this study in which two patients in the CSE group and two patients in the FSNB group required general anesthesia because of failure of the anesthetic technique. These four patients were excluded from the study. Thus, the success of providing adequate anesthesia was $95.2 \%$ (40 patients) in the two groups. There were no significant differences between the two groups in terms of demographic data (sex, weight, and height), American Society of Anesthesiologists (ASA) physical status, site of surgery,

Table 1. Demographic Data

\begin{tabular}{lcc}
\hline & $\begin{array}{c}\text { CSE group } \\
(\mathrm{n}=40)\end{array}$ & $\begin{array}{c}\text { FSNB group } \\
(\mathrm{n}=40)\end{array}$ \\
\hline ASA PS (I/II/III) & $1 / 38 / 0$ & $2 / 36 / 2$ \\
Age (yr) & $67.4 \pm 1.3$ & $71.8 \pm 1.3^{*}$ \\
Sex (M/F) & $4 / 36$ & $3 / 37$ \\
Body weight (kg) & $57.3 \pm 1.5$ & $55.8 \pm 1.7$ \\
Height (cm) & $154.9 \pm 1.2$ & $153.4 \pm 1.5$ \\
Site of surgery (right/left) & $24 / 16$ & $25 / 15$ \\
Preparation time (min) & $9.7 \pm 1.0$ & $11.6 \pm 1.3$ \\
Duration of surgery (min) & $124.5 \pm 4.7$ & $135 \pm 5.9$ \\
Total anesthetic time (min) & $154.4 \pm 3.2$ & $158.6 \pm 2.3$ \\
\hline
\end{tabular}

Values are mean \pm SEM or number of patients. CSE: combined spinal epidural anesthesia, FSNB: femoral/sciatic nerve block with lateral femoral cutaneous nerve block, ASA PS: American Society of Anesthesiologists physical status. ${ }^{*} \mathrm{P}<0.05$ compared with CSE group. preparation time, duration of surgery, and total anesthetic time, though actual age was higher in the FSNB group (67.4 versus 71.8, respectively, $\mathrm{P}=0.018$ ) (Table 1 ).

Intraoperatively, the number of patients who required additional analgesia or sedation using intravenous administration of ketorolac, fentanyl, midazolam, and bolus propofol was significantly higher in the FSNB group compared with the CSE group. However, there were no significant differences between the number of patients requiring propofol infusion, antiemetics, and antihypertensives, except nicardipine. No severe events, such as systemic toxicity of local anesthetics, total spinal anesthesia, or cardiac arrest were reported in any of the patient. There was no difference in frequency of complications between the two groups $(\mathrm{P}>0.05)$. Nevertheless, hypotension (eight patients in the CSE group and four patients in the FSNB group) and bradycardia (four patients in the CSE group and one patient in the FSNB group) occurred in each group. The incidences of each side effect for the postoperative 48 hours are presented in Table 2. No patient had pruritus or sedation postoperatively. No significant differences of the incidences of dizziness and postoperative nausea/vomiting were found between the two groups. Complete resolution time, duration of IV PCA use, and remaining amount of IV PCA were comparable between the two groups (Table 3 ).

No difference was noted between the groups in terms of

Table 2. Postoperative Incidence of Side Effects

\begin{tabular}{llllll}
\hline & \multicolumn{2}{c}{ Dizziness } & & \multicolumn{2}{c}{ PONV } \\
\cline { 2 - 3 } \cline { 5 - 6 } & $\begin{array}{c}\text { CSE } \\
(\mathrm{n}=40)\end{array}$ & $\begin{array}{c}\text { FSNB } \\
(\mathrm{n}=40)\end{array}$ & & $\begin{array}{c}\text { CSE } \\
(\mathrm{n}=40)\end{array}$ & $\begin{array}{c}\text { FSNB } \\
(\mathrm{n}=40)\end{array}$ \\
\hline PO 1 hr & $2(5.0)$ & $0(0.0)$ & & $2(5.0)$ & $1(2.5)$ \\
PO 2 hr & $2(5.0)$ & $0(0.0)$ & & $6(15.0)$ & $5(12.5)$ \\
PO 6 hr & $6(15.0)$ & $5(12.5)$ & & $3(7.5)$ & $1(2.5)$ \\
PO 12 hr & $2(5.0)$ & $1(2.5)$ & & $3(7.5)$ & $3(7.5)$ \\
PO 24 hr & $0(0.0)$ & $1(2.5)$ & & $4(10.0)$ & $4(10.0)$ \\
PO 48 hr & $1(2.5)$ & $1(2.5)$ & & $0(0.0)$ & $0(0.0)$ \\
\hline
\end{tabular}

Values are number of patients (percentage). PONV: postoperative nausea and vomiting, CSE: combined spinal epidural anesthesia, FSNB: femoral/sciatic nerve block with lateral femoral cutaneous nerve block, PO n hr: nth postoperative hour.

Table 3. The Data of Intravenous Patient-Controlled Analgesia

\begin{tabular}{lcc}
\hline & $\begin{array}{c}\text { CSE group } \\
(\mathrm{n}=40)\end{array}$ & $\begin{array}{c}\text { FSNB group } \\
(\mathrm{n}=40)\end{array}$ \\
\hline Duration of IV PCA use (min) & $3,007.1 \pm 153.5$ & $3,596.8 \pm 254.3$ \\
Remaining amount of IV PCA (ml) & $7.5 \pm 3.9$ & $1.4 \pm 0.8$ \\
Complete resolution time (min) & $296.9 \pm 15.7$ & $264.5 \pm 28.4$
\end{tabular}

Values are mean \pm SEM. CSE: combined spinal epidural anesthesia, FSNB: femoral/sciatic nerve block with lateral femoral cutaneous nerve block, IV PCA: intravenous patient-controlled analgesia. 


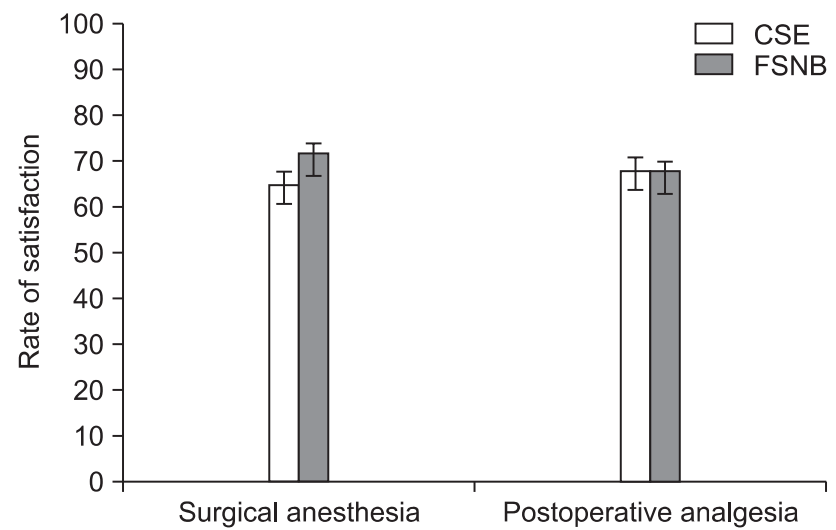

Fig. 1. Rate of satisfaction with surgical anesthesia and postoperative analgesia. Data are expressed as the mean \pm SEM. CSE: combined spinal epidural anesthesia, FSNB: femoral/sciatic nerve block with lateral femoral cutaneous nerve block.

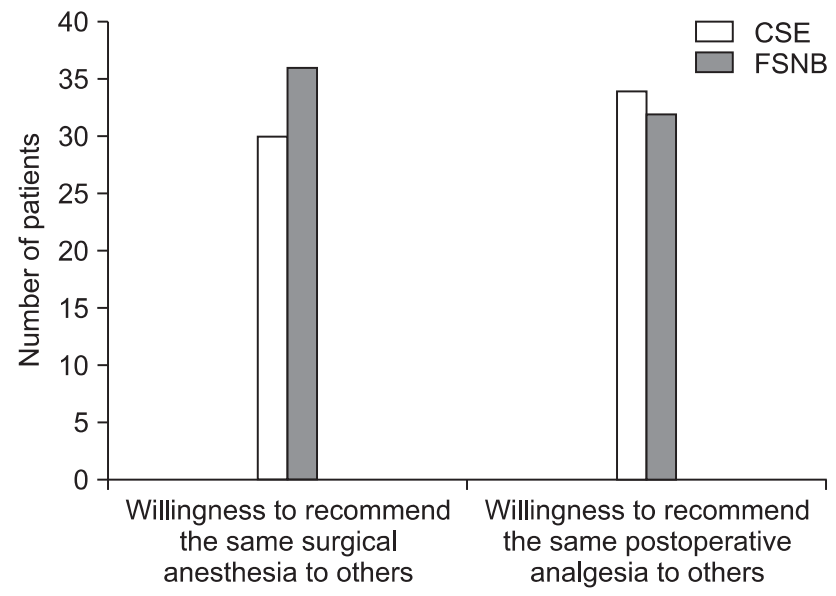

Fig. 2. Numbers of patients who are willing to recommend the same surgical anesthesia and postoperative analgesia. CSE: combined spinal epidural anesthesia, FSNB: femoral/sciatic nerve block with lateral femoral cutaneous nerve block.

the rate of satisfaction with the surgical anesthesia and the postoperative analgesia, and the willingness to recommend the same surgical anesthesia and postoperative analgesia to others (Fig. 1 and 2). There were no significant changes in VAS scores during the postoperative period in each group $(\mathrm{P}>0.05)$. There was also no statistical difference of pain scores between the two groups ( $\mathrm{P}>0.05)$ (Fig. 3).

\section{Discussion}

In the past, lower extremity peripheral nerve blocks were seldom the anesthetic procedure of choice for patients undergoing total joint replacement, primarily because of anesthesiologists' lack of experience in performing these procedures. These blocks are an excellent anesthetic option

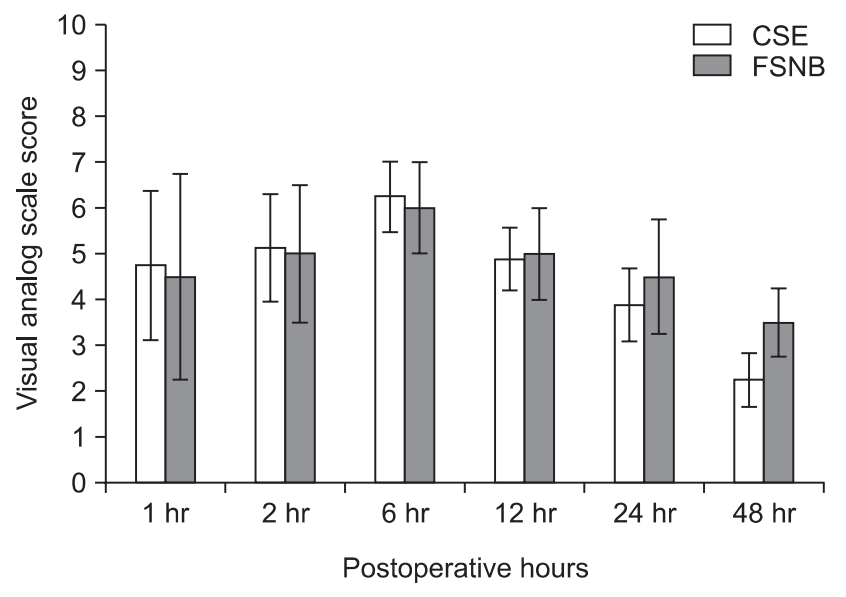

Fig. 3. Visual analog scale score plotted against postoperative hours in the CSE and FSNB groups. Data are expressed as the mean \pm SEM. There are no significant changes in VAS scores during postoperative period in each group, and no significant difference of VAS scores between the two groups. CSE: combined spinal epidural anesthesia, FSNB: femoral/sciatic nerve block with lateral femoral cutaneous nerve block.

for patients who are not candidates for spinal or epidural anesthesia, because septic or anticoagulated patients may safely receive peripheral blocks. Additionally, patients with disease processes that make the hemodynamically significant sympathectomy seen with neuraxial anesthesia unsafe can undergo a lower extremity nerve block without significant risk of hemodynamic instability. Further, severe neurological complications (spinal hematoma, cauda equina syndrome) after central neural blockade are rare events [4] but should be considered when choosing a safe method for surgical anesthesia or postoperative analgesia. Peripheral nerve damage can occur after peripheral nerve block, but the consequences are not as grave [5]. These benefits of lower extremity peripheral nerve block justify its use as an alternative anesthesia in patients undergoing TKRA.

The afferent information of the knee is carried by the lumbar plexus innervating the anterior thigh and medial malleolus, and the sacral plexus innervating the posterior regions of the thigh and knee and some parts of the leg and foot [6]. The lumbar plexus includes the femoral nerve (L2-L4), the obturator nerve (L2-L4), and the lateral femoral cutaneous nerve (L2-L3). And the sacral plexus includes the sciatic nerve [6,7]. Femoral nerve blocks do not reliably cover the obturator nerve [8-10] and addition of obturator nerve blocks improves postoperative analgesia after TKRA [11,12]. Although Eifert et al. [13] showed that $700 \mathrm{mg}$ of mepivacaine without epinephrine in the combined "3-in-1"/sciatic nerve blocks made $3.91 \pm 0.95 \mu \mathrm{g} / \mathrm{ml}$, which is below the threshold for toxic symptoms $(5-6 \mu \mathrm{g} / \mathrm{ml})$, we were reluctant to perform the obturator nerve block in our study where approximately $700 \mathrm{mg}$ of mepivacaine were used 
for FSNB since there are no convincing evidence that dosage over $700 \mathrm{mg}$ is safe from systemic toxicity of local anesthetics.

Accordingly, the quality of surgical anesthesia in the FSNB group was thought to be unreliable in that the use of intraoperative antihypertensives, analgesics, and sedatives was more frequent $(\mathrm{P}<0.05)$ in the FSNB group than in the CSE group, possibly because of an insufficient blockade of the obturator nerve. In some patients in the FSNB group, analgesia was adequate until a specific time, after which discomfort occurred when the surgeon started hammering for the purpose of fitting prosthesis to the bones. Intravenous midazolam and/or propofol with a secure airway were effective in providing sedation in these patients. The slightly higher rate of satisfaction with surgical anesthesia in the FSNB group (though nonsignificantly, $\mathrm{P}=0.078$ ), and no significant difference of the willingness to recommend the same surgical anesthesia to others between the two groups support the effectiveness of the intravenous midazolam and/or propofol in supplementing the insufficient block of the obturator nerve.

Pain after TKRA is marginally treated with IV PCA. After TKRA, VAS scores range from 40-80 (of 100) during the immediate postoperative period and slowly decline by the first operative day [14-16]. In this study, it can be assumed that the effects of singleinjection blockades of the two groups lasted for over 2 hours postoperatively, when total anesthetic time was subtracted from the complete resolution time (165.8 \pm 16.24 in the CSE group and $126.0 \pm 27.70$ in the FSNB group, $\mathrm{P}=0.208$ ). No significant change in postoperative VAS scores suggests that IV PCA provided appropriate analgesia during the period when residual effects of the initial blockades were fading out. In addition, no significant differences in duration of IV PCA use and remaining amount of IV PCA representing the postoperative analgesic consumption, postoperative VAS score of each postoperative hour, the rate of satisfaction with postoperative analgesia, and willingness to recommend the same postoperative analgesia to others between the two groups mean that FSNB achieved a similar degree of the postoperative analgesia in CSE.

The fact that the incidence of intraoperative hypotension and bradycardia is higher in the CSE group than in the FSNB group reflects the more frequent use of ephedrine and atropine in the CSE group than in the FSNB group, though no statistically significant differences of the incidence and the frequency of use were found between groups. Chelly et al. [17] demonstrated that hypotension and bradycardia during surgery was significantly decreased with the use of 3-in-1 paravascular and sciatic blocks compared with epidural anesthesia, and Capdevila et al. [18] also reported a significantly elevated incidence of postoperative arterial hypotension in the continuous epidural infusion group, in comparison with the continuous femoral block group. In addition, the incidence of hypotension was higher in patients receiving epidural block than in patients receiving CSE [19], so it could be explained that relatively better hemodynamic stability in CSE compared with epidural block made incidence of intraoperative hypotension and bradycardia between the two groups in this study statistically indifferent.

The incidence of postoperative side effects, such as dizziness, sedation, postoperative nausea and vomiting (PONV), and pruritus, was similar between the two groups in the present study. Urinary retention, which is one of the most common side effects related to neuraxial blockade, could not be evaluated, because placement of urinary catheters were continued until 48 hours postoperatively in this observational study. Likewise, Davies et al. [20], who did not include urinary retention in the list of side effects, found no significant difference between the two methods. On the contrary, Zaric et al. [21], Singelyn et al. [22], Capdevila et al. [18], and Chelly et al. [17], who included urinary retention in the list of side effects, found less side effects occurred in the patients with a single-shot FSNB or continuous femoral nerve block.

Recently, introduction of ultrasound imaging techniques to peripheral nerve blocks decreases the dose of local anesthetics required to produce a successful surgical block and the time required to perform the procedures, and also provides improvements in onset and success of sensory block. Nevertheless, nerve stimulation should be an essential part of ultrasound guided technique, as ultrasound alone only had a $60 \%$ success rate [23]. Further, ultrasound-aided peripheral nerve stimulated block is perhaps the optimal way to achieve a successful block, especially at the beginning of a learning process [24]. Thus, unavailability of ultrasound at the time of FSNB implies the limitation of our study. By decreasing the dose of local anesthetics required for FSNB under ultrasound guidance, it should have been possible to perform an additional obturator block, which could not be blocked consistently by femoral 3 in 1 block. At this point, we are expecting that using ultrasound imaging techniques in performing FSNB and obturator block in the patient undergoing TKRA will yield more positive results than the present study.

Finally, there are no significant differences of intraoperative and postoperative parameters that we measured except intraoperative use of antihypertensives, analgesics, and sedatives. The results of this study demonstrate that FSNB in addition to a sophisticated use of intraoperative medications, such as sedatives, antihypertensives, and analgesics, provides effective unilateral anesthesia in patients undergoing TKRA as much as CSE. And early postoperative analgesia of single-shot FSNB performed at the beginning of the surgery was also similar to single-shot CSE. However, FSNB cannot be the first choice for TKRA, such as CSE or general anesthesia. It should be used as a third alternative for the situation where CSE or general 
anesthesia cannot be performed. In order to increase efficacy and safety of FSNB for TKRA, further investigations using ultrasound imaging are warranted. In conclusion, FSNB offers a beneficial alternative anesthesia and postoperative analgesia to the patients undergoing TKRA.

\section{References}

1. Rawal N, Van Zundert A, Holmstrom B, Crowhurst JA. Combined spinal-epidural technique. Reg Anesth 1997; 22: 406-23.

2. Green L, Machin SJ. Managing anticoagulated patients during neuraxial anaesthesia. Br J Haematol 2010; 149: 195-208.

3. Howell SJ, Sear YM, Yeates D, Goldacre M, Sear JW, Foex P. Risk factors for cardiovascular death after elective surgery under general anaesthesia. Br J Anaesth 1998; 80: 14-9.

4. Moen V, Dahlgren N, Irestedt L. Severe neurological complications after central neuraxial blockades in sweden 1990-1999. Anesthesiology 2004; 101: 950-9.

5. Fanelli G, Casati A, Garancini P, Torri G. Nerve stimulator and multiple injection technique for upper and lower limb blockade: failure rate, patient acceptance, and neurologic complications. Study Group on Regional Anesthesia. Anesth Analg 1999; 88: 847-52.

6. Allen HW, Liu SS, Ware PD, Nairn CS, Owens BD. Peripheral nerve blocks improve analgesia after total knee replacement surgery. Anesth Analg 1998; 87: 93-7.

7. Hogan MV, Grant RE, Lee L Jr. Analgesia for total hip and knee arthroplasty: A review of lumbar plexus, femoral, and sciatic nerve blocks. Am J Orthop (Belle Mead NJ) 2009; 38: E129-33.

8. Ganidagli S, Cengiz M, Baysal Z, Baktiroglu L, Sarban S. The comparison of two lower extremity block techniques combined with sciatic block: 3-in-1 femoral block vs. psoas compartment block. Int J Clin Pract 2005; 59: 771-6.

9. Bouaziz H, Vial F, Jochum D, Macalou D, Heck M, Meuret P, et al. An evaluation of the cutaneous distribution after obturator nerve block. Anesth Analg 2002; 94: 445-9.

10. Marhofer P, Nasel C, Sitzwohl C, Kapral S. Magnetic resonance imaging of the distribution of local anesthetic during the three-inone block. Anesth Analg 2000; 90: 119-24.

11. McNamee DA, Parks L, Milligan KR. Post-operative analgesia following total knee replacement: an evaluation of the addition of an obturator nerve block to combined femoral and sciatic nerve block. Acta Anaesthesiol Scand 2002; 46: 95-9.

12. Macalou D, Trueck S, Meuret P, Heck M, Vial F, Ouologuem S, et al. Postoperative analgesia after total knee replacement: the effect of an obturator nerve block added to the femoral 3-in-1 nerve block.
Anesth Analg 2004; 99: 251-4.

13. Eifert B, Hahn R, Maier B, Konrad F, Georgieff M. Combined "3-in$1 "$ /sciatic nerve block. Block effectiveness, serum level and side effects using $700 \mathrm{mg}$ mepivacaine $1 \%$ without and with adrenaline and prilocaine 1\%. Anaesthesist 1996; 45: 52-8.

14. Hirst GC, Lang SA, Dust WN, Cassidy JD, Yip RW. Femoral nerve block. Single injection versus continuous infusion for total knee arthroplasty. Reg Anesth 1996; 21: 292-7.

15. Etches RC, Warriner CB, Badner N, Buckley DN, Beattie WS, Chan VW, et al. Continuous intravenous administration of ketorolac reduces pain and morphine consumption after total hip or knee arthroplasty. Anesth Analg 1995; 81: 1175-80.

16. Serpell MG, Millar FA, Thomson MF. Comparison of lumbar plexus block versus conventional opioid analgesia after total knee replacement. Anaesthesia 1991; 46: 275-7.

17. Chelly JE, Greger J, Gebhard R, Coupe K, Clyburn TA, Buckle R, et al. Continuous femoral blocks improve recovery and outcome of patients undergoing total knee arthroplasty. J Arthroplasty 2001; 16: 436-45.

18. Capdevila X, Barthelet Y, Biboulet P, Ryckwaert Y, Rubenovitch J, d'Athis F. Effects of perioperative analgesic technique on the surgical outcome and duration of rehabilitation after major knee surgery. Anesthesiology 1999; 91: 8-15.

19. Rawal N, Schollin J, Wesstrom G. Epidural versus combined spinal epidural block for cesarean section. Acta Anaesthesiol Scand 1988; 32: 61-6.

20. Davies AF, Segar EP, Murdoch J, Wright DE, Wilson IH. Epidural infusion or combined femoral and sciatic nerve blocks as perioperative analgesia for knee arthroplasty. Br J Anaesth 2004; 93 : 368-74.

21. Zaric D, Boysen K, Christiansen C, Christiansen J, Stephensen S, Christensen B. A comparison of epidural analgesia with combined continuous femoral-sciatic nerve blocks after total knee replacement. Anesth Analg 2006; 102: 1240-6.

22. Singelyn FJ, Deyaert M, Joris D, Pendeville E, Gouverneur JM. Effects of intravenous patient-controlled analgesia with morphine, continuous epidural analgesia, and continuous three-in-one block on postoperative pain and knee rehabilitation after unilateral total knee arthroplasty. Anesth Analg 1998; 87: 88-92.

23. Yang WT, Chui PT, Metreweli C. Anatomy of the normal brachial plexus revealed by sonography and the role of sonographic guidance in anesthesia of the brachial plexus. AJR Am J Roentgenol 1998; 171: 1631-6.

24. Orebaugh SL, Williams BA, Kentor ML. Ultrasound guidance with nerve stimulation reduces the time necessary for resident peripheral nerve blockade. Reg Anesth Pain Med 2007; 32: 448-54. 